University of Puget Sound

Sound Ideas

All Faculty Scholarship

Faculty Scholarship

$11-1-1994$

\title{
Entropy of Measurement and Erasure: Szilard's Membrane Model Revisited
}

Harvey S. Leff

Physics Department, California State Polytechnic University, Pomona, 3801 West Temple Avenue, Pomona, California 91768

Andrew F. Rex

University of Puget Sound, rex@pugetsound.edu

Follow this and additional works at: http://soundideas.pugetsound.edu/faculty_pubs

\section{Citation}

Leff, Hs, and Andrew F. Rex. 1994. "Entropy of Measurement and Erasure - Szilards Membrane Model Revisited." American Journal Of Physics 62(11): 994-1000.

This Article is brought to you for free and open access by the Faculty Scholarship at Sound Ideas. It has been accepted for inclusion in All Faculty Scholarship by an authorized administrator of Sound Ideas. For more information, please contact soundideas@pugetsound.edu. 


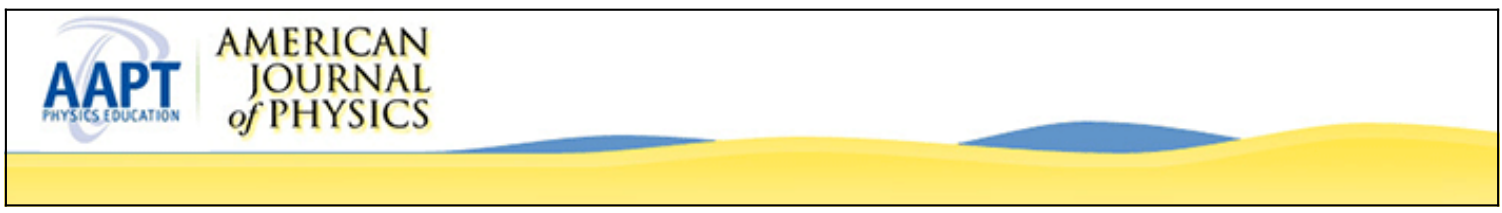

Entropy of measurement and erasure: Szilard's membrane model revisited

Harvey S. Leff and Andrew F. Rex

Citation: American Journal of Physics 62, 994 (1994); doi: 10.1119/1.17749

View online: http://dx.doi.org/10.1119/1.17749

View Table of Contents: http://scitation.aip.org/content/aapt/journal/ajp/62/11?ver=pdfcov

Published by the American Association of Physics Teachers

\section{Articles you may be interested in}

Szilard's Engine: Measurement, Information, and Maxwell's Demon

AIP Conf. Proc. 643, 279 (2002); 10.1063/1.1523817

Goldhaber provided Szilard's isotopes

Phys. Today 54, 14 (2001); 10.1063/1.1420539

The Szilard engine revisited: Entropy, macroscopic randomness, and symmetry breaking phase transitions Chaos 11, 725 (2001); 10.1063/1.1388006

Szilard's inventions patently halted

Phys. Today 54, 102 (2001); 10.1063/1.1366083

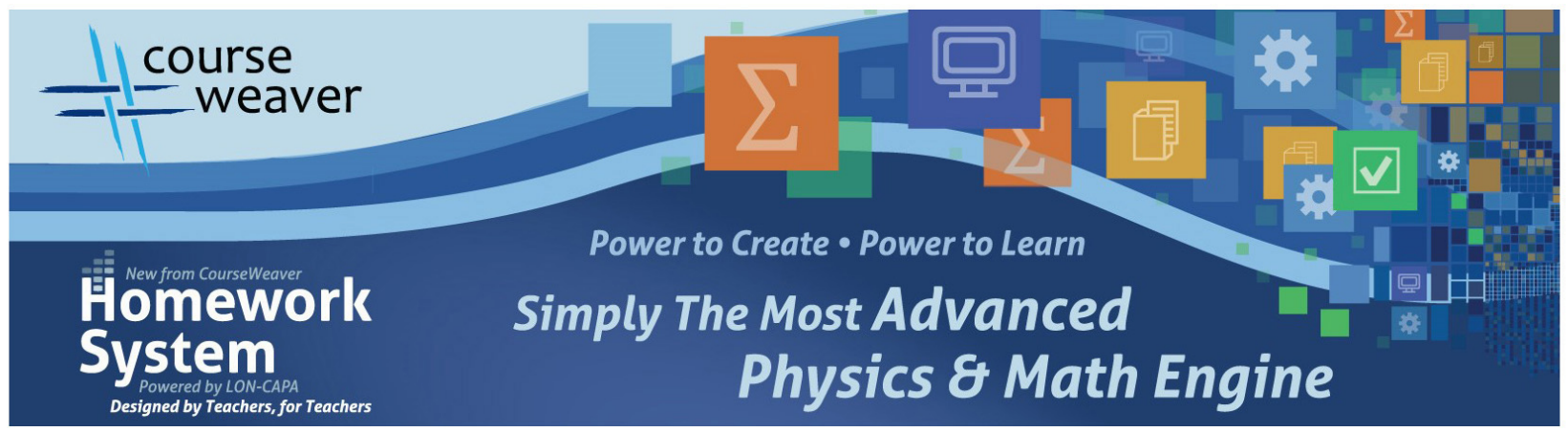




\title{
Entropy of measurement and erasure: Szilard's membrane model revisited
}

\author{
Harvey S. Leffa) \\ California 91768 \\ Andrew F. Rex ${ }^{\text {b) }}$ \\ Physics Department, University of Puget Sound, Tacoma, Washington 98416
}

Physics Department, California State Polytechnic University, Pomona, 3801 West Temple Avenue, Pomona,

(Received 16 February 1994; accepted 18 May 1994)

\begin{abstract}
It is widely believed that measurement is accompanied by irreversible entropy increase. This conventional wisdom is based in part on Szilard's 1929 study of entropy decrease in a thermodynamic system by intelligent intervention (i.e., a Maxwell's demon) and Brillouin's association of entropy with information. Bennett subsequently argued that information acquisition is not necessarily irreversible, but information erasure must be dissipative (Landauer's principle). Inspired by the ensuing debate, we revisit the membrane model introduced by Szilard and find that it can illustrate and clarify (1) reversible measurement, (2) information storage, (3) decoupling of the memory from the system being measured, and (4) entropy increase associated with memory erasure and resetting.
\end{abstract}

\section{INTRODUCTION}

In his Theory of Heat, Maxwell proposed a thought experiment designed to illustrate that the second law of thermodynamics is statistical. ${ }^{1}$ The chief character in that idea became known as Maxwell's demon. ${ }^{2,3}$ For approximately 120 years the puzzle of whether a Maxwell's demon can or cannot violate the second law has been studied and debated. ${ }^{4}$ This rich history inspired a resource letter on Maxwell's demon in this journal. ${ }^{5}$ A resolution based largely on the work of Szilard ${ }^{6}$ and Brillouin ${ }^{7,8}$ gained wide acceptance: $A$ demon must gather information in order to operate, and information acquisition is sufficiently dissipative to "save" the second law. Following the widespread acceptance of this reasoning, it appeared that Maxwell's demon was "dead."

Thirty-one years after Brillouin's work a very different solution to the Maxwell's demon puzzle was proposed by Bennett. ${ }^{9}$ Asserting that information acquisition is not necessarily dissipative, Bennett found Brillouin's resolution to be incomplete. Drawing upon the seminal work of Landauer on energy limits in the computational process, ${ }^{10}$ he proposed an alternative resolution of the puzzle using the notion that a demon requires a memory and must clear that memory periodically. According to Bennett the second law is saved by the fact that erasing and resetting a memory is a dissipative operation.

The importance of memory had been discussed in 1929 by Szilard. ${ }^{6} \mathrm{He}$ introduced the idea of measuring a system parameter $x$ and recording the measurement's result via a corresponding value $y$ in a memory register. He wrote, "Then let $x$ and $y$ be uncoupled after the measurement, so that $x$ can change, while $y$ retains its value for some time. Such measurements are not harmless interventions. A system in which such measurements occur shows a sort of memory faculty, in the sense that one can recognize by the state parameter $y$ what value another state parameter $x$ had at an earlier moment, and we shall see that simply because of such a memory the second law would be violated, if the measurement could take place without compensation."

Szilard identified both "intervention" and "memory" as key ingredients, providing seeds for Brillouin's and Bennett's subsequent work. One of Szilard's three examples, which involved a heat engine with a one-molecule working fluid, became quite popular and was examined further by a variety of researchers. Expounding on that model in his book
Foundations of Statistical Mechanics, Penrose ${ }^{11}$ noted that memory erasure is dissipative. He wrote, "The large number of distinct observational states that the Maxwell demon must have in order to make significant entropy reductions possible may be thought of as a large memory capacity in which the demon stores the information about the system which he acquires as he works reducing its entropy. As soon as the demon's memory is completely filled, however, ... he can achieve no further reduction of the Boltzmann entropy. He gains nothing, for example, by deliberately forgetting or erasing some of his stored information in order to make more memory capacity available; for the erasure, being a setting process, itself increases the entropy by an amount at least as great as the entropy decrease made possible by the newly available memory capacity."

Bennett's subsequent ideas regarding irreversible memory erasure and also the possibility of reversible information acquisition were developed independently, and have received enthusiastic support. ${ }^{12-14}$ Unfortunately, a difficulty with Bennett's thesis is that relatively few known thermodynamical models clearly illustrate nondissipative information acquisition. Identification of unambiguous examples would help resolve criticisms ${ }^{4}$ of the subtle points involved.

Our purpose here is to re-examine a simple, tractable membrane model introduced by Szilard in 1929 along with his one-molecule gas model. In contrast with the latter model, which has been discussed widely, the membrane model has received little attention. We show that a simple modification of his analysis can shed light on the issue of information acquisition and erasure. The modified membrane model is related to the Maxwell's demon puzzle in the sense that it takes a working fluid through a cyclic process during which: (a) physical measurements (which are reversible in this case) are made, (b) the results are stored in a memory register, (c) the memory is then decoupled from the working fluid, and (d) the memory register is erased and reset irreversibly.

Szilard's membrane model differs from the Maxwell's demon problem in that it does not involve conversion of heat to work. Although it is too specialized to allow general conclusions, it clearly illustrates the central points that link the efforts of Szilard, Landauer, and Bennett. In Secs. II and III the original form of the model is revisited, and two questionable parts of Szilard's analysis are scrutinized. In Sec. IV we 
introduce a simplified variant of Szilard's model that succinctly illustrates information acquisition, storage, and erasure. Section V contains a discussion of our main results.

\section{SZILARD'S MEMBRANE MODEL}

The Szilard membrane model ${ }^{6}$ consists of an $\mathrm{N}$-molecule dilute gas contained in a volume $V$. This system is in thermal contact with an energy reservoir at temperature $T$. The gas molecules can exist in either of two forms, labeled " 1 " and " 2 ," and transitions $1 \leftrightarrow 2$ can be induced over relatively long time intervals (relative to typical observation times) via interactions with neighboring gas molecules. A real physical example is provided by molecular hydrogen, which can exist as ortho-hydrogen, with total nuclear spin $=1$ (and $z$ components $-1,0,+1$ ) or as para-hydrogen, with total nuclear spin $=0$. Equilibration times for hydrogen are on the order of years. This example was mentioned in Ehrenberg's 1967 Scientific American article on Maxwell's demon. ${ }^{15}$

Interactions between species 1 and 2 are assumed to be sufficiently weak that the fluid behaves as a mixture of two ideal gases. The internal energy of the gas can be written

$$
U_{\mathrm{gas}}=U_{1}(T)+U_{2}(T),
$$

where each term is of the form,

$$
U_{i}(T)=N_{i} e_{i}(T) \text { for } i=1,2 .
$$

$e_{i}(T)$ is the average energy per molecule for species $i, N_{i}$ is the molecule number for this species, and $N_{1}+N_{2}=N$. In thermodynamic equilibrium the average species fractions are $w_{1}(T)$ and $w_{2}(T)$, with $w_{1}(T)+w_{2}(T)=1$, and $0<w_{i}(T)<1$ for $i=1,2$, i.e.,

$$
N_{i} \equiv N_{i}(T) \equiv w_{i}(T) N
$$

for $i=1,2$.

The gas entropy is

$$
S_{\mathrm{gas}}=S_{1}+S_{2} \text {, }
$$

where each single-component entropy has the ideal gas form $^{16}$

$$
S_{i}=N_{i} k \ln \left[f_{i}(T) V / N_{i}\right] \text { for } i=1,2 .
$$

$f_{1}$ and $f_{2}$ can differ from one another (as can $e_{1}$ and $e_{2}$ ) but they are functions only of $T$, and the argument of the logarithm is dimensionless. Szilard did not write down Eqs. (1), (2), and (4) explicitly, but they are very useful. In what follows we adopt the shorthand notation $w_{1}=w_{1}(T)$ and $w_{2}=w_{2}(T)$.

The container is assumed to have two telescoping sections, $A A^{\prime}$ and $B^{\prime} B$ as illustrated in Fig. 1. Initially, the sections are telescoped together [Fig. 1(a)] giving the (minimum possible) volume $V$, the gas is in thermal equilibrium, and $N_{i}=w_{i} N$ for $i=1,2$. End piece $A^{\prime}$ of section $A A^{\prime}$ is a semipermeable membrane that passes only species 1 molecules; and end piece $B^{\prime}$ of $B^{\prime} B$ is permeable only to species 2 molecules. Although real semipermeable membranes are not perfect sorters, Szilard assumed ideal membranes that are. We retain this assumption throughout this article. Szilard prescribed a four-step cyclic process that entails information acquisition, storage of information in a memory register, decoupling of the system and memory register, and erasure of the information. In Sec. III we describe his steps, provide some details omitted from his paper, and suggest two modifications to make his analysis more useful. (a)

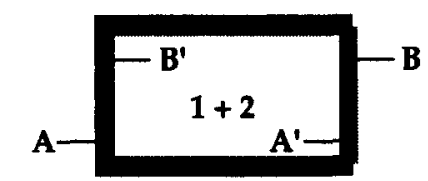

(b)

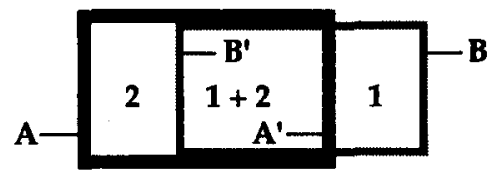

(c)

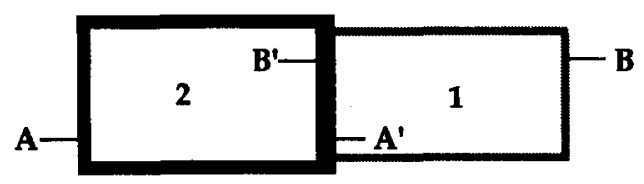

Fig. 1. (a) Gas species 1 and 2 before step 2. (b) Two containers telescoping outward during step 2 , with membrane $B^{\prime}$ permeable only to species 2 and membrane $A^{\prime}$ permeable only to species 1 . (c) The configuration upon completion of step 2 , which separates species 1 and 2 .

\section{SZILARD'S CYCLIC PROCESS}

\section{A. Step 1: Measurement}

Each molecule of type 1 is assigned an $x$ value of 1 , and each one of type 2 is assigned an $x$ value of 2 . Szilard envisioned "intervention" by an intelligent being, who measures the $x$ values of each molecule at some initial time, and assigns the value $y=1$ to all molecules with $x=1$ and the value $y=2$ to all molecules with $x=2$. The set $\{y\}$ is remembered by the intelligent being, and does not change even if a molecule is later transformed by interactions with other molecules and/or with the container walls, from type 1 to type 2 or vice versa. In such events the molecule's $x$ value changes but its $y$ value does not. The main point is: Each molecule's $y$ value represents its initial species type.

It is assumed that these measurements of the $y$ variables do not change the thermodynamic state of the gas; i.e., the entropy change of the gas is

$$
\left[\Delta S_{\text {gas }}\right]_{\text {step } 1}=0 \text {. }
$$

On the other hand, Szilard proposed that the intervention needed to determine the set $\{y\}$ generates a positive entropy change,

$$
\left[\Delta S_{\text {intervention }}\right]_{\text {step } 1}>0 \text {. }
$$

Szilard did not discuss the mechanism for this entropy increase, but assumed that there were no other entropy changes in the environment (the parts of the universe other than the gas and intervention mechanism); i.e.,

$$
\left[\Delta S_{\text {env }}\right]_{\text {step } 1}=0 \text {. }
$$

Given Eqs. (5a) and (5c), evidently the intervention entropy in Eq. (5b) must be an entropy increase of the intervening intelligent being itself. 


\section{B. Step 2: Separation}

After the measurement intervention, the two container sections are telescoped outward, leading from the configuration in Fig. 1(a) to that in Fig. 1(c). The total volume increases from $V$ to $2 V$. We assume this is done slowly enough to keep the process quasistatic, but fast enough that $N_{1}$ and $N_{2}$ are unchanged. Because of the semipermeable membranes $A$ and $B$, all type-1 molecules are moved into the resulting right chamber and all type- 2 molecules go into the left chamber [Fig. 1(c)]. The ideal membranes are assumed to sort perfectly and to do zero work on the species they transmit. Zero net external work is done on both species because $A$ and $A^{\prime}$ do equal and opposite work on species 2 and $B^{\prime}$ and $B$ do equal and opposite work on species 1.

The internal energy of each component is unchanged because $U_{i}=w_{i} N e_{i}(T)$ for $i=1,2$; the temperature $T$ does not vary; and during the time interval of step 2 , neither do $N_{1}$ and $N_{2}$. Thus, there is zero net heat transfer between the gas and reservoir. Telescoping is a reversible process, so inward telescoping will recover the original thermodynamic state as long as $N_{1}$ and $N_{2}$ do not vary. It follows from Eqs. (3) and (4) that

$$
\left[\Delta S_{\text {gas }}\right]_{\text {step } 2}=0 .
$$

Szilard regarded both the intervention mechanism and the environment as having no thermodynamic involvement during separation, i.e.,

$$
\left[\Delta S_{\text {intervention }}\right]_{\text {step } 2}=0,
$$

and

$$
\left[\Delta S_{\text {env }}\right]_{\text {step } 2}=0,
$$

though he did not show all these entropy changes explicitly.

\section{Step 3: Equilibration}

After separation, neither of the gases in the two storage chambers is in thermal equilibrium. That is, the $\{x\}$ set in each chamber changes over time while the $\{y\}$ set remains fixed. Specifically, just after separation, $N_{1}=w_{1} N$ and $N_{2}=0$ in the right chamber, and $N_{2}=w_{2} N$ and $N_{1}=0$ in the left chamber. After a sufficiently long time interval, $\left(N_{1}\right)_{\text {right }} \rightarrow w_{1}^{2} N$ and $\left(N_{2}\right)_{\text {right }} \rightarrow w_{2} w_{1} N$, while $\left(N_{1}\right)_{\text {left }} \rightarrow w_{1} w_{2} N$ and $\left(N_{2}\right)_{\text {left }} \rightarrow w_{2}^{2} N$. This equilibration constitutes the third step of the cycle.

Szilard assumed that equilibration occurs at constant temperature, and thus the internal energy per molecule is unchanged for each species. Further, because the overall molecular fractions of the species do not change during equilibration, neither do $U_{1}$ and $U_{2}$ even though the energy in each chamber changes (if $e_{1} \neq e_{2}$ ). Therefore after equilibration, $\quad\left(N_{1}\right)_{\text {total }}=\left(N_{1}\right)_{\text {left }}+\left(N_{1}\right)_{\text {right }} \rightarrow w_{1} w_{2} N+w_{1}^{2} N=w_{1} N$, $\left(N_{2}\right)_{\text {total }}=\left(N_{2}\right)_{\text {left }}+\left(N_{2}\right)_{\text {right }} \rightarrow w_{2}^{2} N+w_{1} w_{2} N=w_{2} N, \quad$ and $U_{i}=w_{i} N e_{i}(T)$ for $i=1,2$. Equation (4) implies that during equilibration the entropy change in the left chamber is $\Delta S_{\text {left equil }}=w_{2} N k\left[w_{1} \ln \left(f_{1} / f_{2}\right)-w_{1} \ln w_{1}-w_{2} \ln w_{2}\right] ; \quad$ the entropy change in the right chamber is $\Delta S_{\text {right equil }}$ $=w_{1} N k\left[-w_{2} \ln \left(f_{1} / f_{2}\right)-w_{1} \ln w_{1}-w_{2} \ln w_{2}\right]$. Adding these gives

$$
\left[\Delta S_{\text {gas }}\right]_{\text {step } 3}=-N k\left\{w_{1} \ln w_{1}+w_{2} \ln w_{2}\right\}>0 .
$$

Because the intervention mechanism is not involved,

$$
\left[\Delta S_{\text {intervention }}\right]_{\text {step } 3}=0 \text {. }
$$

Szilard wrote, "If we allow this equilibrium to be achieved in both containers independently and at constant volume and temperature, then the entropy of the system certainly has increased." He went on to say, "If we accomplish the equilibrium distribution in both containers in a reversible fashion then the entropy of the rest of the world will decrease by the same amount." Accordingly, he found an entropy decrease for the environment of

$$
\begin{aligned}
{\left[\Delta S_{\text {env }}\right]_{\text {step } 3} } & =-\left[\Delta S_{\text {gas }}\right]_{\text {step } 3} \\
& =N k\left\{w_{1} \ln w_{1}+w_{2} \ln w_{2}\right\}<0 .
\end{aligned}
$$

Szilard wrote down an equation equivalent to Eq. (7c), but his assumption of reversibility is curious, for it is difficult to envision reversal of the equilibration process. We return to this point later.

\section{Step 4: Cycle completion}

Seeking a zero-work process to remix the gases in a single volume $V$, Szilard specified that in step 4, membranes $A^{\prime}$ and $B^{\prime}$ are to be replaced with ones permeable only to molecules with $y=1$ and $y=2$, respectively. This is an interesting concept, for it requires an ability to sort molecules based upon remembrance of a previous physical state. It transcends the abilities of real membranes, which sort on the basis of the present molecular states. One might interpret the required Szilard membranes to be collections of gates that are operated by the intervener of step 1 , who remembers the $y$ value for each molecule. With this view we cautiously follow Szilard's lead.

With the $y$-selector membranes in place, the sections are telescoped inward, reducing the total volume from $2 V$ to $V$ isothermally, doing zero work (as before) in the process. Equation (2) implies zero internal energy change for the gas because $N_{1}$ and $N_{2}$ do not change, and $T$ is constant. Equations (3) and (4) imply the entropy change

$$
\left[\Delta S_{\text {gas }}\right]_{\text {step } 4}=+N k\left\{w_{1} \ln w_{1}+w_{2} \ln w_{2}\right\}<0 .
$$

Because the intervention mechanism is used here, it appears that

$$
\left[\Delta S_{\text {intervention }}\right]_{\text {step } 4}>-N k\left\{w_{1} \ln w_{1}+w_{2} \ln w_{2}\right\} .
$$

If, as implied by Szilard's discussion, the entropy of the environment is unaltered,

$$
\left[\Delta S_{\text {env }}\right]_{\text {step } 4}=0 \text {. }
$$

Szilard expounded on what step 4 accomplishes: "The distribution of the $y$-coordinate ... now has become statistically independent of the $x$-values and besides we are able to reestablish the original distribution ... (of the $y$ values). Thus we would have gone through a complete cycle. The only change that we have to register is the resulting decrease of entropy ... [given by Eq. (8a)]." Szilard seemed to be acknowledging two things: a cyclic process for the working fluid and memory erasure-namely, re-establishment of the original $y$ distribution.

\section{E. Implications of the whole cycle}

Szilard utilized the fact that for the gas cycle, $\left[\Delta S_{\text {gas }}\right]_{\text {cycle }}=0$, and the net entropy change of the environment is the sum of Eqs. $(5 \mathrm{c}),(6 \mathrm{c}),(7 \mathrm{c})$, and $(8 \mathrm{c})$ :

$$
\left[\Delta S_{\text {env }}\right]_{\text {cycle }}=N k\left\{w_{1} \ln w_{1}+w_{2} \ln w_{2}\right\}<0 .
$$






Fig. 2. A graphical depiction of relation (12), $\exp \left(-\Delta s_{1} / k\right)+\exp \left(-\Delta s_{2} / k\right) \leqslant 1$. For any $\Delta s_{2}$, the value of $\Delta s_{1}$ must lie above the solid curve, in the shaded region.

Recalling that Szilard did not account for intervention in step 4, Eqs. (5b), (6b), (7b), and (9a) along with the second law imply the total entropy change,

$$
\begin{aligned}
& {\left[\Delta S_{\text {intervention }}\right]_{\text {step } 1}+\left[\Delta S_{\text {env }}\right]_{\text {cycle }}} \\
& \quad=\left[\Delta S_{\text {intervention }}\right]_{\text {step } 1}+N k\left\{w_{1} \ln w_{1}+w_{2} \ln w_{2}\right\} \geqslant 0 .
\end{aligned}
$$

This is his first major result for the membrane model.

Szilard then assumed that the intervention entropy could be written

$$
\left[\Delta S_{\text {intervention }}\right]_{\text {step } 1}=N_{1} \Delta s_{1}+N_{2} \Delta s_{2},
$$

where $\Delta s_{i}$, the entropy change associated with the measurement of a species $i$ molecule, is independent of the probabilities $w_{1}$ and $w_{2}$. Combining Eqs. (9b) and (10),

$$
w_{1} \Delta s_{1}+w_{2} \Delta s_{2}+k\left\{w_{1} \ln w_{1}+w_{2} \ln w_{2}\right\} \geqslant 0 \text {. }
$$

Minimizing the left-hand side with respect to $w_{1}\left(=1-w_{2}\right)$ for constant $\Delta s_{1}$ and $\Delta s_{2}$ gives $^{17}$

$$
\exp \left(-\Delta s_{1} / k\right)+\exp \left(-\Delta s_{2} / k\right) \leqslant 1,
$$

which is Szilard's second major result. The inequality (12) means that the intervention entropy pairs $\left(\Delta s_{1}, \Delta s_{2}\right)$ must lie in the shaded region of Fig. 2.

The above description of Szilard's membrane model suggests two places where the analysis can be improved. The first is in step 3, where Szilard used the thermodynamic reversibility condition $\Delta S_{\text {gas }}+\Delta S_{\text {env }}=0$. There is no compelling reason to impose this reversibility condition because the irreversible equilibration does not require any entropy change in the environment. The analysis can be improved by replacing the reversibility assumption (7c) by the condition,

$$
\left[\Delta S_{\text {env }}\right]_{\text {step } 3}=0 .
$$

Second, Szilard's omission of the intervention device in step 4 is questionable. The gas mixtures in the right and left chambers must be combined into the final volume $V$, incur- ring the negative entropy change for the gas given by Eq. (8a), which was not shown in Szilard's paper. To satisfy the second law a compensating entropy increase must exist somewhere. This cannot be in the environment because: $N_{1}$ and $N_{2}$ are unaltered, the process is isothermal, there is zero change in the internal energies $U_{1}$ and $U_{2}$, zero work is done on the gas, and thus the first law of thermodynamics implies zero heat transfer to the environment; i.e., Eq. (8c) holds. Evidently step 4 cannot be accomplished without an entropy increase associated with intervention, as given in Eq. (8b).

For completeness, we observe that one might imagine attempting the inward telescoping in step 4 using membranes $A^{\prime}$ and $B^{\prime}$ that are one-way filters, which allow molecules to cross membrane $B^{\prime}$ from left to right only and membrane $A^{\prime}$ from right to left only. However, these would presumably be subject to thermal fluctuations associated with Smoluchowski-type trapdoors, enabling molecules to actually move in both directions. ${ }^{18,19}$ This again supports the conclusion that the use of the intervener's memory is unavoidable for Szilard's step 4, and that the entropy increase in Eq. (8b) is unavoidable.

Incorporating our two improvements, we replace Eq. (7c) with Eq. (13), add the entropy changes in parts (a), (b), and (c) of Eqs. (5) - (8), and apply the second law to get

$$
\begin{gathered}
{\left[\Delta S_{\text {intervention }}\right]_{\text {step } 1}+\left[\Delta S_{\text {intervention }}\right]_{\text {step } 4}} \\
>-N k\left\{w_{1} \ln w_{1}+w_{2} \ln w_{2}\right\} .
\end{gathered}
$$

The entropy increase in steps 1 and 4 can be understood as follows. In step 1, molecules are examined by the Maxwell's demon and their $y$ values are recorded in a memory. In step 4 , as molecules approach the set of gates described earlier, the Maxwell's demon must again examine each molecule and check against its memory to see if its $y$ variable is 1 or 2 . This again entails recording results in a memory, even if only temporarily.

These acts increase the entropy of the demon's memory as its memory addresses take on values other than their initial standardized values (say all zeros). In essence the memory goes from all zeros to a mixture of zeros and ones. If we repeat this experiment with many such similar systems and memories, the resulting ensemble of memories will have a nonzero statistical entropy. Presumably, this entropy increases with the number of measurements made until the memories are full and information must be erased in order to continue. We conclude that the results of the cyclic process are: (i) an environment with a lowered entropy, and (ii) a (jumbled) demon's memory whose entropy is higher than it was initially. Erasure of this memory would reduce its entropy to zero (in its standard state) but would send a compensating entropy to the environment in accordance with Landauer's principle. ${ }^{10}$

Our two modifications of Szilard's cycle are intended to clarify how that cycle can be interpreted in terms of our current understanding of measurement, memory, and memory erasure. In Sec. IV, we consider a variant of Szilard's membrane model that illustrates these same features in an even simpler and more direct way.

\section{VARIATION ON SZILARD'S THEME}

Reflection on the Szilard cycle described above suggests the use of an even simpler cycle that clearly illustrates four important elements: reversible measurement, storage in a memory, decoupling of the memory from the system, and 




Fig. 3. Modified Szilard membrane cycle, which consists of: separation and measurement (step $2^{\prime}$ ), equilibration (and decoupling, step $3^{\prime}$ ), and erasure +resetting (step $4^{\prime}$ ). Note that after separation the total number of molecules in the left and right chambers are different if $w_{1} \neq w_{2}$.

entropy increase associated with memory erasure and resetting. We describe this variant in terms of the modified steps $1^{\prime}, 2^{\prime}, 3^{\prime}$, and $4^{\prime}$, as illustrated in Fig. 3.

\section{A. Step 1': Nothing}

This is no step at all because we omit intervention by the intelligent being that is specified in Szilard's step 1. This omission is possible because measurement is accomplished in step $2^{\prime}$.

\section{B. Step 2': Separation and measurement}

This is the same as Szilard's step 2, namely a slow outward telescoping of the containers. However we now interpret the membrane actions as constituting "measurements" of the $x$ values of the molecules. That is, if the $i$ th molecule passes through $A^{\prime}$, it has $x_{i}=1$, and if the $j$ th molecule passes through $B^{\prime}$, it has $x_{j}=2$. The separation process stores molecules with initial values of $x=1$ and 2 in separate chambers. Together, these chambers can be regarded as a memory register that stores information about the original $x$ variable for each molecule. In essence the separation process results in the measurement of $\left(x_{1}, x_{2}, \ldots, x_{N}\right)$ and the correspondence: $\left(x_{1}, x_{2}, \ldots, x_{N}\right) \Rightarrow\left(y_{1}, y_{2}, \ldots, y_{N}\right)$ during the separation process: If molecule $i$ is in the right (or left) chamber, then $y_{i}=1$ (or 2). Of course

$$
\left[\Delta S_{\text {gas }}\right]_{\text {step } 2^{\prime}}=\left[\Delta S_{\text {env }}\right]_{\text {step } 2^{\prime}}=0 .
$$

\section{Step 3': Equilibration}

Equilibration of the species is irreversible, and work can be done by neighboring molecules on one another to induce changes, say, in nuclear spin states. We assume however that zero net work is done on the gas by the container walls during equilibration. The entropy change of the gas in step $3^{\prime}$ is identical with Eq. (7a):

$$
\left[\Delta S_{\text {gas }}\right]_{\text {step } 3^{\prime}}=-N k\left\{w_{1} \ln w_{1}+w_{2} \ln w_{2}\right\}>0 .
$$

Constancy of the internal energy and zero net external work imply zero net heat transfer and zero entropy change for the environment. Therefore, Eq. (7c) must be replaced by the equivalent of Eq. (13),

$$
\left[\Delta S_{\text {env }}\right]_{\text {step } 3^{\prime}}=0 \text {. }
$$

\section{Step 4': Memory erasure and resetting}

It is not possible to use a real membrane to bring both gases into the single volume $V$ with a zero external work process. Therefore we define step $4^{\prime}$ to consist of two parts: first, removal of the central partitions $A^{\prime}$ and $B^{\prime}$, so that the gases mix in volume $2 V$; and second, reversible, isothermal compression of the mixed gas from volume $2 V$ to volume $V$. In the first part there is no internal energy change, but there is an entropy change,

$$
\left[\Delta S_{\text {gas }}\right]_{\text {erasure }}=N k\left[w_{1} \ln w_{1}+w_{2} \ln w_{2}+\ln 2\right],
$$

which is positive if $w_{1} \neq w_{2}$ and zero if $w_{1}=w_{2}$. Equation (17a) is the entropy of erasure, which can be given a useful interpretation based upon Landauer' ${ }^{20}$ view of erasure: the separate phase spaces of the gases in chambers 1 and 2 "diffuse into one another" irreversibly (unless $w_{1}=w_{2}$, in which case partition removal gives zero entropy change and partition replacement recovers the original state). During the erasure there is zero entropy change in the environment:

$$
\left[\Delta S_{\text {env }}\right]_{\text {erasure }}=0 \text {. }
$$

Resetting the memory by a reversible isothermal compression requires an external work $W>0$ on the gas and a concomitant heat transfer $Q<0$ ( $t o$ the gas). The first law of thermodynamics demands that $\Delta U=Q+W=0$, or $Q=-W$ $=N k T \ln 2$, and the corresponding entropy change of the constant-temperature environment is

$$
\left[\Delta S_{\text {env }}\right]_{\text {resetting }}=-\left[\Delta S_{\text {gas }}\right]_{\text {resetting }}=N k \ln 2 .
$$

Adding Eqs. (17a) and (17c) gives the overall entropy change for the gas during step 4',

$$
\left[\Delta S_{\text {gas }}\right]_{\text {step } 4^{\prime}}=N k\left[w_{1} \ln w_{1}+w_{2} \ln w_{2}\right]<0 .
$$

Adding the entropy changes in Eqs. (17b)-(17d), the total entropy change for the erasure/resetting combination is seen to be

$$
\begin{aligned}
{\left[\Delta S_{\text {gas +env }}\right]_{\text {erasure/resetting }} } & \\
& =\left[\Delta S_{\text {gas }}\right]_{\text {step } 4^{\prime}}+\left[\Delta S_{\text {env }}\right]_{\text {step } 4^{\prime}} \\
& =N k\left[w_{1} \ln w_{1}+w_{2} \ln w_{2}+N k \ln 2\right] \geqslant 0 .
\end{aligned}
$$

The evident non-negativity of Eq. (18) is consistent with the second law.

\section{E. Implications of the whole cycle}

The foregoing enables verification that $\left[\Delta S_{\text {gas }}\right]_{\text {cycle }}=0$ (as it must) and

$$
\left[\Delta S_{\text {env }}\right]_{\text {cycle }}=N k \ln 2 .
$$

If we make the association

$$
\left[\Delta S_{\text {env }}\right]_{\text {cycle }} \equiv N\left[w_{1} \Delta s_{1}+w_{2} \Delta s_{2}\right]=N k \ln 2,
$$

it is clear that if $\Delta s_{1}=\Delta s_{2} \equiv \Delta s$, then $\Delta s=k \ln 2$, independent of the values of $w_{1}$ and $w_{2}$. Here $\Delta s_{1}$ and $\Delta s_{2}$ should be viewed as the entropies of erasure (per molecule) rather than entropies of measurement. Further if we adopt Szilard's assumption that $\Delta s_{1}$ and $\Delta s_{2}$ are in fact independent of $w_{1}$ and $w_{2}$, then the only possible entropies of erasure are those for which $\Delta s_{1}=\Delta s_{2}=k \ln 2$ because in Eq. (20), $w_{1} \Delta s_{1}+w_{2} \Delta s_{2}=w_{1}\left(\Delta s_{1}-\Delta s_{2}\right)+\Delta s_{2}$ must equal $k \ln 2$ independent of $w_{1}$. Thus the strict equality in Eq. (12) holds: $\exp \left(-\Delta s_{1} / k\right)+\exp \left(-\Delta s_{2} / k\right)=1$. Evidently this means that the erasure of each $y$ value is accompanied by an entropy 
increase $k \ln 2$ in the environment. This can be associated with the loss of information available about the (binary) $y$ values.

\section{DISCUSSION}

In Sec. IV we have simplified Szilard's membrane model by omitting use of an intelligent being to make measurements, recognizing that the action of the semipermeable membranes effects both reversible measurement and memory storage, and observing that memory erasure and resetting requires work by an external agent. Prior to the erasure step, there exists a record of the initial $y$ variables - namely, the numbers of molecules in the left and right chambers. After erasure no record of the initial $y$ distribution remains.

An important characteristic of the membrane model is the irreversible equilibration that occurs after separation of the two species. This process destroys the correlation between the sets $\{x\}$ and $\{y\}{ }^{21}$ We can verify this by examining the joint probability $P(x, y)$ of finding a molecule in species state $x$ and with the memory value $y$, where $x$ and $y$ can take the values 1 or 2 . We do this both before and after the equilibration following the separation step. Just after separation and before equilibration, there is perfect correlation between the $x$ and $y$ variables on both sides. In the left side, $P_{\text {left }}(x, y)=\delta_{x y} \delta_{y 2}$ and on the right side, $P_{\text {right }}(x, y)=\delta_{x y} \delta_{y 1}$, where $\delta_{x y}$ is the Kronecker delta, which $=1$ for $x=y$, and 0 for $x \neq y$. Information entropies associated with these distributions can be defined via $I_{q}=-k \Sigma_{x} \Sigma_{y} P_{q}(x, y) \ln P_{q}(x, y)$, where $q=$ left or right. Because of the perfect $x-y$ correlation and the restriction that $x=y=(1,2)$ on the (right, left) prior to equilibration, it follows that $I_{q}=0$ for $q=$ left and right just after separation. This means there is zero missing information. The species type of each molecule is fixed, and also known from the chamber (left or right) in which it resides.

After equilibration is complete, the joint probabilities are $P_{\text {left }}(x, y)=w_{x} \delta_{y 2}$ and $P_{\text {right }}(x, y)=w_{x} \delta_{y 1}$. The corresponding information entropies are $I_{\text {left }}=I_{\text {right }}$ $=-k\left[w_{1} \ln w_{1}+w_{2} \ln w_{2}\right]$. This can be interpreted as the entropy per molecule associated with the $x$ and $y$ variables after equilibration. The information entropy differences for step 3 are $\Delta I_{\text {left }}=\Delta I_{\text {right }}=-k\left[w_{1} \ln w_{1}+w_{2} \ln w_{2}\right]$. Notice that $\Delta I_{\text {left }}$ and $\Delta I_{\text {right }}$ are not quite the same as $\Delta S_{\text {left equil }}$ and $\Delta S_{\text {right equil }}$ in the discussion preceding Eq. (7a) for two reasons. First, $\Delta I$ does not include temperature-dependent effects, but is restricted to configurational changes and second, $\Delta I$ is a per-molecule information entropy change. Because the fraction of molecules on the left is $w_{2}$ and that on the right is $w_{1}$, we may write the total information entropy change associated with the $x$ and $y$ variables as

$$
\begin{aligned}
\Delta I_{x-y} \text { equilibration } & =N w_{1} \Delta I_{\text {right }}+N w_{2} \Delta I_{\text {left }} \\
& =-N k\left[w_{1} \ln w_{1}+w_{2} \ln w_{2}\right],
\end{aligned}
$$

which is identical with Eqs. (7a) and (16a). This equivalence shows that the irreversible equilibration process generates entropy solely because of the destruction of the cor elations between the $x$ and $y$ variables. In other words, dezoupling the memory from the system is an entropy-producing process that brings uncertainty about the species type of each molecule.

In our discussion of Szilard's original cycle, we observed that the memory gains entropy when information is recorded within it. A similar entropy increase occurs in the memory of a Maxwell's demon as it gathers information. Such entropy increases are best viewed in an ensemble sense: If many similar demons measure many similar systems, the resulting ensemble of memories has an entropy that is greater than the zero initial entropy when each ensemble member's memory was in a known reference state. The modified membrane model is very different because every species 1 (or 2) molecule is in the right (or left) chamber after the measurement and separation step. Separation yields no uncertainties other than those associated with fluctuations in the fractions $w_{1}$ and $w_{2}$. Indeed we showed above that the entropy change of the gas and environment were both zero during separation.

Although there is something troubling about filling a memory without generating a concomitant entropy change, this situation is only temporary. During the slow process of species equilibration, entropy is generated as the memory variables $\{y\}$ become decoupled from the gas variables $\{x\}$. Of course the memory remains intact while this happens. The entropy of decoupling, Eq. (16a), plays the role of an "entropy of memory" in that it results from the dual process of recording measurements of certain system variables in a memory and then decoupling that memory from those variables. It is certainly not an entropy of acquisition because it is generated long after the measurement step. The final resetting process transfers both the entropy of decoupling, Eq. (16a), and the entropy of erasure, Eq. (17a), to the environment. In essence the cycle transfers energy from one part of the environment (work source) to another part (constanttemperature reservoir). Its value lies not in what it accomplishes as a physical device, but in how it can help us learn about information acquisition, storage, and erasure.

We close with a remark about Leo Szilard. It is a tribute to his ingenuity that after 65 years, the clever models he invented are still providing food for thought and tools for understanding.

\section{ACKNOWLEDGMENT}

We thank Rolf Landauer for his helpful comments on a first draft of this article.

a)E-mail: hsleff@csupomona.edu
b)E-mail: rex@ups.edu
1J. C. Maxwell, Theory of Heat (Longmans, London, 1871), Chap. 12.
${ }^{2}$ W. Thomson, "The kinetic theory of the dissipation of energy," Nature 9,
$441-444$ (1874). Reprinted in Lord Kelvin's Mathematical and Physical
Papers (Cambridge University Press, Cambridge, 1911), Vol. 5, pp. 11-
20. Reprinted in Ref. 2.
${ }^{3}$ W. Thomson, "The sorting demon of Maxwell," R. Inst. Proc. 9, 113-114
(1879). Reprinted in Lord Kelvin's Mathematical and Physical Papers in
Ref. 2, pp. 21-23

${ }^{4}$ H. S. Leff and A. F. Rex, Maxwell's Demon: Entropy, Information, Computing (Princeton University Press, Princeton, and Adam Hilger/IOP, Great Britain, 1990).

${ }^{5}$ H. S. Leff and A. F. Rex, "Resource letter: Maxwell's demon," Am. J. Phys. 58, 201-209 (1990).

${ }^{6} \mathrm{~L}$. Szilard, "On the decrease of entropy in a thermodynamic system by the intervention of intelligent beings," Z. Phys. 53, 840-856 (1929); in B. T. Feld and G. Weiss Szilard, The Collected Works of Leo Szilard: Scientific Papers (MIT Press, Cambridge, 1972), pp. 103-129, English translation is by A. Rapoport and M. Knoller; reprinted in J. A. Wheeler and W. H. Zurek, Quantum Theory and Measurement (Princeton University Press, Princeton, 1983), pp. 539-548, reprinted also in Ref. 4.

${ }^{7}$ L. Brillouin, "Maxwell's demon cannot operate: Information and entropy.

I," J. Appl. Phys. 22, 334-337 (1951). Reprinted in Ref. 4.

${ }^{8}$ L. Brillouin, Science and Information Theory (Academic Press, New York, 1956), Chap. 13. 
${ }^{9} \mathrm{C}$. H. Bennett, "The thermodynamics of computation-a review," Int. J. Theor. Phys. 21, 905-940 (1982). Reprinted in Ref. 4.

${ }^{10} \mathrm{R}$. Landauer, "Irreversibility and heat generation in the computing process," IBM J. Res. Dev. 5, 183-191 (1961). Reprinted in Ref. 4.

${ }^{11}$ O. Penrose, Foundations of Statistical Mechanics (Pergamon Press, Oxford, 1970), pp. 221-238.

${ }^{12} \mathrm{R}$. Landauer, "Information is physical," Phys. Today 44 (5), 23-29 (May 1991).

${ }^{13} \mathrm{~J}$. D. Barrow, The World Within the World (Oxford University Press, Oxford, 1988), pp. 127-130.

${ }^{14}$ W. H. Zurek, "Maxwell's demon, Szilard's engine and quantum measurements," in G. T. Moore and M. O. Scully, Frontiers of Nonequilibrium Statistical Physics (Plenum Press, New York, 1984), pp. 1 and 161. Reprinted in Ref. 4.

${ }^{15}$ W. Ehrenberg, "Maxwell's demon," Sci. Am. 217 (5), 103-110 (November 1967).

${ }^{16}$ This follows from the fact that the canonical partition function for classical ideal gases has the form $Z=\left[g V\left(2 \pi m k T / h^{2}\right)^{3 / 2} q_{\text {rot }} q_{\text {vib }} q_{\mathrm{el}}\right]^{N} / N$ !, where $m$ is the molecular mass, $h$ is Planck's constant, $g$ is the spin degeneracy, and the $q$ factors are the single-molecule partition functions for rotational, vibrational, and electronic transitions, respectively. Each $q$ depends only on $T$ and internal properties of the molecules; see for example, D. S. Betts and R. E. Turner, Introductory Statistical Mechanics (Addison-Wesley, Reading, MA, 1992), pp. 125-129.

${ }^{17}$ Setting the derivative of the left side of Eq. (11) equal to zero gives $w_{2}=w_{1} \exp \left(\left[\Delta s_{1}-\Delta s_{2}\right] / k\right)$ and $w_{1}=1 /\left[1+\exp \left(\left[\Delta s_{1}-\Delta s_{2}\right] / k\right)\right.$. Substituting back into Eq. (11) yields, $w_{1}\left[\Delta s_{1} / k+\ln w_{1}\right]$ $\times\left[1+\exp \left(\left[\Delta s_{1}-\Delta s_{2}\right] / k\right)\right] \geqslant 0$, which reduces to Eq. (12).

${ }^{18}$ P. A. Skordos and W. H. Zurek, "Maxwell's demon, rectifiers, and the second law: Computer simulation of Smoluchowski's trapdoor," Am. J. Phys. 60, 876-882 (1992).

${ }^{19}$ A. F. Rex and R. Larsen, "Entropy and information for an automated Maxwell's demon," in Workshop on Physics and Computation PhysComp '92 (IEEE Computer Society Press, Los Alamitos, 1993), pp. 93-101.

${ }^{20} \mathrm{R}$. Landauer, "Information is physical," in Ref. 19, pp. 1-4.

${ }^{21} \mathrm{~A}$ related analysis is given by M. J. Klein, "Note on a problem concerning the Gibbs paradox," Am. J. Phys. 26, 80-81 (1958). A gas with its nuclei in an excited metastable state and another with the same type nuclei in the ground state, are mixed. As one species decays into the other, the entropy of mixing "disappears" but there is a compensating entropy increase in a constant temperature reservoir. In contrast, our present example unmixes (separates) the gases first and the subsequent equilibration induces new mixing because the single species gases each become two-species gases.

\title{
On the resistance between two points on a grid
}

\author{
Giulio Venezian \\ Physics Department, Southeast Missouri State University, Cape Girardeau, Missouri 63701
}

(Received 8 November 1993; accepted 6 June 1994)

\begin{abstract}
The resistance between two adjacent nodes on an infinite square grid of equal resistors can easily be found by superposition. This paper addresses the corresponding problem for two arbitrary nodes. A solution is found by exploiting the symmetry of the grid and using the method of superposition. The mathematical problem involves the solution of an infinite set of linear, inhomogeneous difference equations which are solved by the method of separation of variables.
\end{abstract}

\section{INTRODUCTION}

A recently published general physics textbook ${ }^{1}$ presents a problem in which the student is asked to find the resistance between two adjacent nodes in an infinite plane grid of identical resistors. As a follow-up question, the student is then asked to find the resistance between two nodes which are an arbitrary distance apart. From the statement of the problem, it is apparent that the author of the problem assumed that the second question could be answered as easily as the first. In a private communication with the writer of this article, the author of the problem confirmed that he had made that assumption. ${ }^{2}$ The second problem turns out to be considerably more complicated than the first. It requires the application of mathematical techniques which are beyond the reach of the average general physics student, but would make it a good problem to use in a mathematical physics class to show an application of two-dimensional difference equations, a topic that is often neglected.

\section{DESCRIPTION OF THE PROBLEM}

The physical situation is illustrated in Fig. 1. An infinite number of identical resistors of resistance $R$ are connected to form a square grid. The problem is to find the resistance that would be measured between two arbitrarily spaced nodes. The basic approach used here is the same as that used by Paul $^{3}$ to treat the case of adjacent nodes. For this reason, the problem of adjacent nodes will be discussed first.

\section{ADJACENT RESISTORS}

Following Paul or Purcell, ${ }^{3}$ for instance, let a current $I$ enter the grid at a node $P$ and let it come out of the grid at a distant point. If the return point is removed to infinity, the problem is invariant under $90^{\circ}$ rotations, so the current flowing through each of the four resistors connected to node $P$ will be equal. Thus each of the four resistors carries a current equal to $I / 4$. The resulting voltage drop between node $P$ and an adjacent node $Q$ will be equal to $(I / 4) R$, the product of the resistance of the resistor and the current passing through it.

Now consider the problem of a current $I$ entering the grid at a distant point and exiting at the adjacent node, $Q$. In this case, the current pattern at $Q$ will be symmetric and the current flowing from $P$ to $Q$ will be $I / 4$, and the voltage drop from $P$ to $Q$ will again be given by $(I / 4) R$. 